\title{
Colorectal cancer screening with fecal occult blood test: A 22-year cohort study
}

\author{
PENG JIN $^{1,2}$, ZI-TAO WU ${ }^{1}$, SHI-RONG LI ${ }^{1}$, SHU-JUN LI ${ }^{1}$, JI-HENG WANG ${ }^{1}$, ZHI-HONG WANG $^{1}$, \\ JIAN-GUO LU ${ }^{1}$, XIN-JUAN CUI ${ }^{1}$, YING HAN ${ }^{1}$, JIANYU RAO ${ }^{3,4}$ and JIAN-QIU SHENG ${ }^{1}$ \\ ${ }^{1}$ Department of Gastroenterology, Beijing Military General Hospital, Beijing 100700; \\ ${ }^{2}$ Third Military Medical University, Chongqing 400038, P.R. China; ${ }^{3}$ Department of Pathology and Laboratory Medicine, \\ David Geffen School of Medicine; ${ }^{4}$ Department of Epidemiology, School of Public Health, \\ University of California, Los Angeles, CA 90015, USA
}

Received December 23, 2012; Accepted May 30, 2013

DOI: $10.3892 / 01.2013 .1402$

\begin{abstract}
The aim of the present study was to investigate the efficacy of colorectal cancer (CRC) screening with a three-tier fecal occult blood test (FOBT) in the Chinese population. The study was performed between 1987 and 2008 at the Beijing Military General Hospital, in a cohort of army service males and females aged $>50$ years. Between 1987 and 2005, a three-tier screening program, comprising guaiac-based FOBTs (gFOBTs), followed by immunochemical FOBTs for positive guaiac test samples and then colonoscopy for positive immunochemical test subjects, was performed annually. The cohort was followed up until 2008. The cohort included 5,104 subjects, of which, 3,863 subjects participated in screening (screening group) and 1,241 did not (non-screening group). The two groups did not differ in age, gender or other major risk factors for colon cancer. Overall, 36 CRCs occurred in the screening group and 21 in the non-screening group. Compared with the non-screening group, the relative risk for the incidence and mortality of CRC was 0.51 [95\% confidence interval (CI), 0.30-0.87] and 0.36 (95\% CI, 0.18-0.71), respectively, in the screening group. The general sensitivity of this three-tier FOBT was $80.6 \%$ (95\% CI, 65.3-91.1). Thus, annual screening using the three-tier FOBT program may reduce the CRC incidence and mortality rate.
\end{abstract}

\section{Introduction}

Colorectal cancer (CRC) is the second most common type of cancer and the second leading cause of cancer-related mortality in developed countries (1). In recent years, the incidence of CRC has markedly increased in China, particularly in urban populations. This is likely to be due to the rapid changes

Correspondence to: Professor Jian-Qiu Sheng, Department of Gastroenterology, Beijing Military General Hospital, 5 Nanmenchang, Dongcheng, Beijing 100700, P.R. China E-mail: jianqiu@263.net

Key words: colorectal cancer, screening, fecal occult blood test in social economic factors, such as lifestyle, diet and environment. As an example, in Beijing, the annual incidence of CRC has increased from 16 per 100,000 to 24 per 100,000 in the past decade (2).

Screening provides the ability to detect precancerous lesions and early-stage $\mathrm{CRC}$, thus saving lives, and there are numerous different screening options presently available (3). Among these, the fecal occult blood test (FOBT) is the most common test in use for CRC screening. Several large cohort studies have demonstrated the effectiveness of FOBT-based screening in Western countries (4-6); however, no such studies have been performed in developing countries thus far. Furthermore, the majority of the large-scale cohort studies have utilized the conventional chemical guaiac-based FOBT (gFOBT) method. The gFOBT has a high false-positive rate (range, 2-13\%) $(7,8)$ and other limitations, such as the requirement of diet restriction. The latter is particularly troublesome for people in Asian countries. The antibody-based immunochemical method, the fecal immunochemical test (FIT), has been demonstrated to have test performance characteristics with improved sensitivity and specificity compared with those of gFOBT, and it does not require any restrictions on diet (7). However, the higher costs associated with FIT compared with gFOBT limit its use for screening in countries such as China, where healthcare resources are limited. The present study evaluates a three-tier FOBT-based program, and presents the results of a prospective longitudinal trial for CRC screening with this program.

\section{Materials and methods}

Subjects. The eligible subjects in the present study were army officers (retired or non-retired) aged $>50$ years, who were receiving healthcare from the Beijing Military General Hospital (Beijing, China) and lived in the Beijing area. Subjects with known CRC, colorectal adenomas, inflammatory bowel disease or various types of malignant tumors were excluded from the cohort. The study was approved by the Beijing Military General Hospital Ethics Committee, and informed consent was obtained from each subject.

The present study was a dynamic cohort study, where subjects who met the eligibility criteria were recruited on an 
annual basis. Upon entry into the cohort study, each subject received a complete health status check-up, which included a physical examination, chest X-ray, ECG, abdominal ultrasound, mammogram (for females) and serological examination, including an analysis of glucose and lipid levels. The health check-up data, along with the data from a baseline questionnaire (for date of birth, gender, education, family history of malignant tumors, lifestyle, medication use, body height/weight and previous general health status), were entered into a database. The database was updated yearly, based on the findings from the annual follow-up examinations.

At the end of the initial check-up, each eligible subject was asked by physicians for their consent to undergo CRC screening. Those who agreed to be screened were included in the screening group, and those who refused to be screened were included in a non-screening group. Other than the FOBT that was performed in the screening group on an annual basis, the subjects in the screening and non-screening groups were investigated in the same manner, with an annual examination for other chronic conditions, as detailed above.

The annual FOBT-based CRC screening began in May 1987 and ended in December 2005. Following this, the two groups were followed up until December 2008. In 1987, there were 3,002 eligible subjects, including 2,260 males and 742 females, with a mean age of 61.8 years $(2,809$ subjects were aged $\leq 74$ years). The number of subjects in the screening group was 2,246, and the number of subjects in the non-screening group was 756. For the subsequent years (1988-2005), an additional 2,102 eligible subjects were entered into the cohort. In total, there were 3,863 subjects in the screening group and 1,241 in the non-screening group (Fig. 1).

Screening methods. For the screening, each subject provided one fecal sample every year for the FOBT, which was performed in the Clinical Laboratory of Beijing Military General Hospital. No restrictions, with regard to diet, drugs or other food items, were imposed on the subjects prior to stool collection. Between 1987 and 2005, a three-tier screening program was implemented: gFOBT (rehydrated; produced by Baso Diagnostics Inc., Zhuhai, China) was performed, followed by FIT (gold gel stripe; provided by WanhuaPuman Biological Engineering Co., Ltd., Tech Lt. Comp., Beijing, China) for gFOBT-positive fecal samples, and then colonoscopy for those who had positive FIT results. According to the manufacturer, the positive threshold of FIT was $0.2 \mu \mathrm{g} /$ ml. For patients who refused colonoscopy, a double-contrast barium enema (DCBE) was performed. For patients with negative (no cancer detected) colonoscopy or DCBE results, the aforementioned screening steps were repeated in the following year.

On identification of a lesion (polyp, tumor or otherwise) during colonoscopy or DCBE, a biopsy (or resection for a polyp or tumor) was performed. If a malignant tumor was identified, appropriate management was provided according to the final pathology, and the subject was regarded as an incidence case (the secondary endpoint) and continued to be followed up until they succumbed to the disease (the primary endpoint). Patients with adenoma were to be followed annually, as were the other non-cancer subjects, following complete resection.

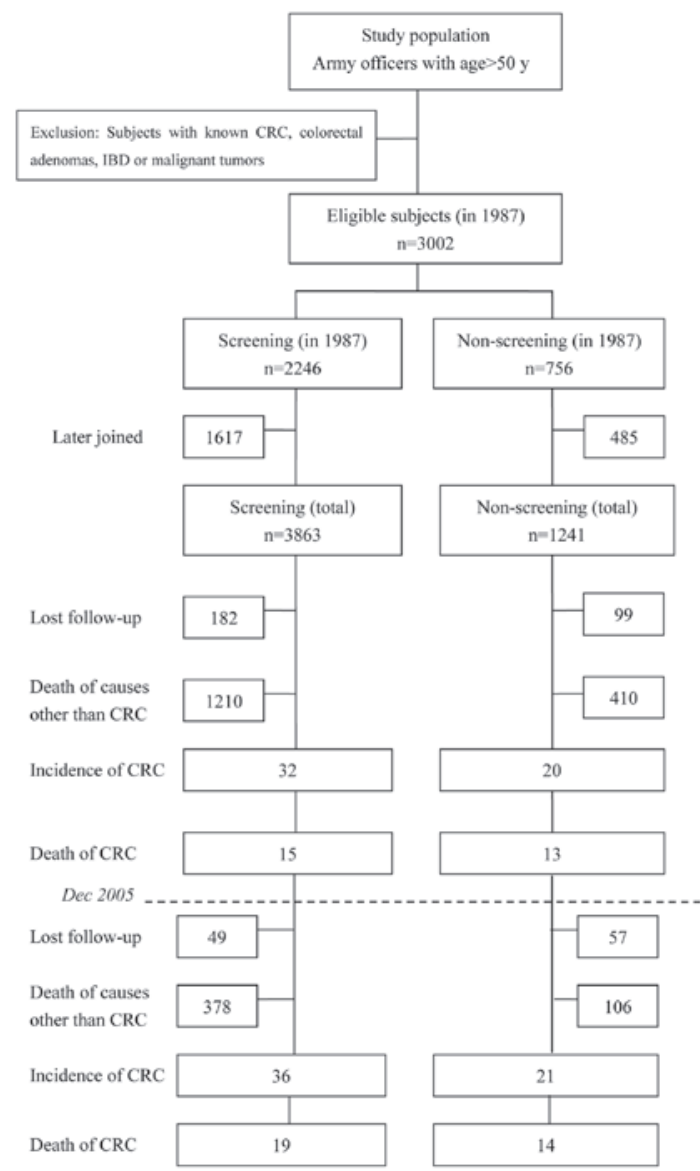

Figure 1. Diagram depicting the screened versus non-screened population and their outcomes.

Data and statistical analyses. The primary endpoint of the follow up was CRC-related mortality, and the secondary endpoint was the incidence of CRC. Overall, $82 \%$ of the mortalities occurred in the Beijing Military General Hospital, and the cause was determined based on a review of the medical records (International Classification of Diseases, ICD-9 or -10). For the subjects who succumbed elsewhere, a telephone interview with the next of kin was performed and the cause of death was determined from the death certificate.

A positive predictive value (PPV) was estimated for each screening round, while only positive tests followed by colonoscopy or DCBE were used in the computation. The general sensitivity was evaluated by the number of true positives relative to the number of individuals with carcinomas, while a positive test result was considered to be a true positive if a carcinoma was detected during the entire follow-up period.

To evaluate potential selection bias, the $\chi^{2}$ test or the independent samples t-test for the baseline characteristics [age at baseline, gender, education, family history of malignant tumors, body mass index (BMI), smoking status (never, former or current), alcohol intake (none, occasional or regular), physical exercise (at least once per week or less), meat intake (at least three times per week or less) and aspirin use (regular or never)] and status at time of mortality from all of these causes were utilized. A $\chi^{2}$ test was used to examine the CRC incidence and mortality in the screening and non-screening groups, and the relative risk (RR) and 95\% confidence intervals (CIs) were 
Table I. Baseline characteristics of subjects in the screening and non-screening groups.

\begin{tabular}{|c|c|c|c|}
\hline Characteristics & Screening $(n=3863)$ & Non-screening $(n=1241)$ & P-value \\
\hline \multicolumn{4}{|l|}{ Gender, n (\%) } \\
\hline Male & $2913(75.4)$ & $934(75.3)$ & \multirow[t]{2}{*}{0.917} \\
\hline Female & $950(24.6)$ & $307(24.7)$ & \\
\hline \multicolumn{4}{|l|}{ Age (years) } \\
\hline Mean age \pm SD & $62.1 \pm 6.9$ & $62.0 \pm 7.0$ & 0.613 \\
\hline \multicolumn{4}{|l|}{ Education, n (\%) } \\
\hline Up to high school & $3549(91.9)$ & $1133(91.3)$ & \multirow[t]{2}{*}{0.523} \\
\hline College or higher & $314(8.1)$ & $108(8.7)$ & \\
\hline \multicolumn{4}{|c|}{ Family history of malignant tumors, $\mathrm{n}(\%)$} \\
\hline With & $118(3.1)$ & $31(2.5)$ & \multirow[t]{2}{*}{0.311} \\
\hline Without & 3745 (96.9) & $1210(97.5)$ & \\
\hline \multicolumn{4}{|l|}{ Body mass index } \\
\hline Mean BMI \pm SD & $23.1 \pm 1.6$ & $23.2 \pm 1.6$ & 0.736 \\
\hline \multicolumn{4}{|l|}{ Smoking status, $\mathrm{n}(\%)$} \\
\hline Never & $1339(34.7)$ & $406(32.7)$ & \multirow[t]{3}{*}{0.082} \\
\hline Former & $1120(29.0)$ & $401(32.3)$ & \\
\hline Current & $1404(36.3)$ & $434(35.0)$ & \\
\hline \multicolumn{4}{|l|}{ Alcohol intake, n (\%) } \\
\hline None & $747(19.3)$ & $244(19.7)$ & \multirow[t]{3}{*}{0.630} \\
\hline Occasional & $2810(72.7)$ & 909 (73.2) & \\
\hline Regular & $306(7.9)$ & $88(7.1)$ & \\
\hline \multicolumn{4}{|c|}{ Physical exercise, n (\%) } \\
\hline$<$ Once per week & $1853(48.0)$ & $593(47.8)$ & \multirow[t]{2}{*}{0.910} \\
\hline$\geq$ Once per week & $2010(52.0)$ & $648(52.2)$ & \\
\hline \multicolumn{4}{|l|}{ Meat intake, n (\%) } \\
\hline$<3$ times per week & $1038(26.9)$ & $331(26.7)$ & \multirow[t]{2}{*}{0.891} \\
\hline$\geq 3$ times per week & $2825(73.1)$ & $910(73.3)$ & \\
\hline \multicolumn{4}{|l|}{ Aspirin, n (\%) } \\
\hline No use & $2957(76.5)$ & $958(77.2)$ & \multirow[t]{2}{*}{0.638} \\
\hline Regular use & $906(23.5)$ & $283(22.8)$ & \\
\hline
\end{tabular}

calculated. The Kaplan-Meier survival analysis was used to evaluate the effect of screening on CRC incidence and mortality, and the cumulative incidence and cumulative mortality curves were determined. The Cox proportional hazards regression model was used to control potential confounding factors (age at baseline, gender, education, family history of malignant tumors, BMI, smoking status, alcohol intake, physical exercise, meat intake and aspirin use) for CRC incidence and mortality. SPSS 13.0 software (SPSS, Inc., Chicago, IL, USA) was used for the statistical analyses. All statistical tests that were performed were two-sided, and $\mathrm{P}<0.05$ was considered to indicate a statistically significant difference.

\section{Results}

Subjects and screening results. Fig. 1 shows a diagram depicting the screened vs. non-screened population and the outcome of the study. For the entire 22-year study period, there were a total of 5,104 eligible subjects. Of these, 3,863 participated in the screening for CRC (screening group) and 1,241 did not (non-screening group). Over the course of the study, 231 subjects in the screening group and 156 in the non-screening group were lost to follow-up due to a change in residence (6.0 and $12.6 \%$, respectively; $\mathrm{P}=0.000$ ). In addition, 1,588 subjects in the screening group and 516 in the non-screening group had succumbed due to causes other than CRC (41.1 and $41.6 \%$, respectively; $\mathrm{P}=0.769$ ). Up to December, 2008, the person-years of observation in the screening and non-screening groups were 49,566 and 15,826, respectively. The baseline characteristics of the subjects were not significantly different in the screening group compared with those in the non-screening group (Table I).

Table II shows the results of each screening round. The positive result rates of gFOBT ranged from 4.6 to $16.2 \%$, while FIT ranged from 1.1 to $2.6 \%$. Between 1987 and 2005, 778 colonoscopies and 33 DCBEs were performed in the screening group, with a total follow-up rate of $87.0 \%$ (811/932) in patients with positive FIT results. A total of 36 cases of CRC occurred in the screening group. Among the $36 \mathrm{CRC}$ cases, 25 were detected by the screening program, while four cases with positive FIT results refused to undergo follow-up colonoscopy or DCBE, and were 
Table II. Results of each annual screening round and the test performance of FOBT.

\begin{tabular}{|c|c|c|c|c|c|c|c|c|c|c|c|c|c|}
\hline \multirow[b]{2}{*}{ Year } & \multirow{2}{*}{$\begin{array}{c}\text { No. of } \\
\text { subjects }\end{array}$} & \multicolumn{2}{|c|}{$\begin{array}{l}\text { Screened } \\
\text { subjects }\end{array}$} & \multicolumn{2}{|c|}{$\mathrm{gFOBT}^{+}$} & \multicolumn{2}{|c|}{$\mathrm{FIT}^{+}$} & \multicolumn{2}{|c|}{$\begin{array}{l}\text { Colonoscopy } \\
\text { or DCBE }\end{array}$} & \multicolumn{2}{|c|}{ Cancer } & \multicolumn{2}{|c|}{$\begin{array}{c}\text { Cancer and } \\
\text { adenomas } \geq 1 \mathrm{~cm}\end{array}$} \\
\hline & & $\mathrm{n}$ & $\%$ & $\mathrm{n}$ & $\%$ & $\mathrm{n}$ & $\%$ & $\mathrm{n}$ & $\%^{\mathrm{a}}$ & $\mathrm{n}$ & $\mathrm{PPV}^{\mathrm{b}}$ & $\mathrm{n}$ & $\mathrm{PPV}^{\mathrm{b}}$ \\
\hline 1987 & 2246 & 2246 & 100.0 & 202 & 9.0 & 51 & 2.3 & 45 & 88.2 & 1 & 2.2 & 10 & 22.2 \\
\hline 1988 & 2239 & 2233 & 99.7 & 175 & 7.8 & 48 & 2.1 & 39 & 81.3 & 1 & 2.6 & 8 & 20.5 \\
\hline 1989 & 2240 & 2228 & 99.5 & 237 & 10.6 & 52 & 2.3 & 47 & 90.4 & 2 & 4.3 & 8 & 17.0 \\
\hline 1990 & 2211 & 2205 & 99.7 & 156 & 7.1 & 47 & 2.1 & 35 & 74.5 & 0 & 0.0 & 7 & 20.0 \\
\hline 1991 & 2184 & 2164 & 99.1 & 233 & 10.8 & 52 & 2.4 & 45 & 86.5 & 1 & 2.2 & 12 & 26.7 \\
\hline 1992 & 2286 & 2253 & 98.6 & 254 & 11.3 & 55 & 2.4 & 49 & 89.1 & 2 & 4.1 & 8 & 16.3 \\
\hline 1993 & 2293 & 2285 & 99.7 & 216 & 9.5 & 54 & 2.4 & 46 & 85.2 & 1 & 2.2 & 11 & 23.9 \\
\hline 1994 & 2264 & 2251 & 99.4 & 365 & 16.2 & 58 & 2.6 & 53 & 91.4 & 2 & 3.8 & 10 & 18.9 \\
\hline 1995 & 2308 & 2302 & 99.7 & 269 & 11.7 & 61 & 2.6 & 57 & 93.4 & 3 & 5.3 & 15 & 26.3 \\
\hline 1996 & 2280 & 2247 & 98.6 & 271 & 12.1 & 54 & 2.4 & 51 & 94.4 & 2 & 3.9 & 11 & 21.6 \\
\hline 1997 & 2220 & 2214 & 99.7 & 204 & 9.2 & 52 & 2.3 & 46 & 88.5 & 1 & 2.2 & 7 & 15.2 \\
\hline 1998 & 2217 & 2201 & 99.3 & 316 & 14.4 & 50 & 2.3 & 44 & 88.0 & 1 & 2.3 & 12 & 27.3 \\
\hline 1999 & 2255 & 2249 & 99.7 & 198 & 8.8 & 53 & 2.4 & 39 & 73.6 & 1 & 2.6 & 9 & 23.1 \\
\hline 2000 & 2253 & 2236 & 99.2 & 215 & 9.6 & 52 & 2.3 & 50 & 96.2 & 1 & 2.0 & 14 & 28.0 \\
\hline 2001 & 2232 & 2218 & 99.4 & 192 & 8.7 & 48 & 2.2 & 43 & 89.6 & 1 & 2.3 & 5 & 11.6 \\
\hline 2002 & 2289 & 2273 & 99.3 & 183 & 8.1 & 45 & 2.0 & 41 & 91.1 & 1 & 2.4 & 11 & 26.8 \\
\hline 2003 & 2457 & 2415 & 98.3 & 112 & 4.6 & 26 & 1.1 & 21 & 80.8 & 1 & 4.8 & 6 & 28.6 \\
\hline 2004 & 2457 & 2445 & 99.5 & 227 & 9.3 & 48 & 2.0 & 36 & 75.0 & 2 & 5.6 & 6 & 16.7 \\
\hline 2005 & 2456 & 2431 & 99.0 & 145 & 6.0 & 26 & 1.1 & 24 & 92.3 & 1 & 4.2 & 8 & 33.3 \\
\hline
\end{tabular}

${ }^{\text {aR }}$ ates were the number of colonoscopy or double-contrast barium enema (DCBE) patients relative to the number of patients with positive fecal immunochemical test (FIT) results. 'Only positive tests followed by colonoscopy or DCBE were used in the computation for positive predictive value (PPV). gFOBT, guaiac-based fecal occult blood test.

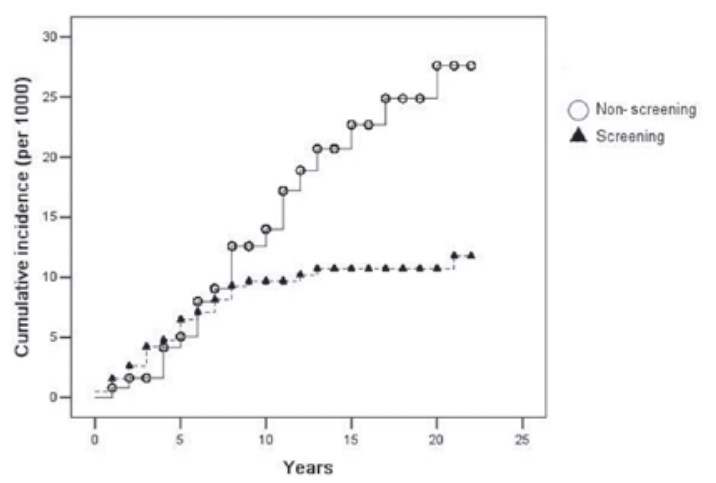

Figure 2. Cumulative incidence of colorectal cancer (CRC) in the screening and non-screening group by Kaplan-Meier analysis.

later diagnosed by clinical means. Seven cases were missed due to a false-negative FOBT result. The general sensitivity of this three-tier FOBT was $80.6 \%$ (29/36; 95\% CI, 65.3-91.1). There were $153(4.0 \%)$ subjects who were identified to have adenomas of $\geq 1 \mathrm{~cm}$ in size in the screening group.

Incidence and mortality rates of CRC in screening and non-screening groups. During the 19-year screening period (1987-2005), 32 CRC cases occurred in the screening group and 20 occurred in the non-screening group. In the last 3 years of follow-up (2006-2008), an additional four cases occurred in the screening group and one occurred in the non-screening group (Fig. 1 and Table III). Until December 2005, the CRC incidence in the screening group was $0.75 / 1,000$ person-years, which was significantly lower than that of the non-screening group $(1.43 / 1,000$ person-years; $\mathrm{P}=0.027)$. The mortality was $0.35 / 1,000$ person-years in the screening group, which was also significantly lower than that of the non-screening group $(0.93 / 1,000, \mathrm{P}=0.013)$. The relative risks of incidence and mortality adjusted for the baseline characteristics of the subjects were 0.49 (95\% CI, 0.28-0.85) and 0.31 (95\% CI, 0.14-0.65), respectively (Both $\mathrm{P}<0.05$; Table III). Three years subsequent to the termination of the screening program, the incidence and mortality in the screening group was significantly lower than that of the non-screening group $(\mathrm{P}=0.034$ and $\mathrm{P}=0.022$, respectively), with adjusted relative risks of 0.51 (95\% CI, 0.30-0.87) and 0.36 (95\% CI, 0.18-0.71), respectively (both $\mathrm{P}<0.05$; Table III). Therefore, there was a $49 \%$ decrease in incidence and a $64 \%$ decrease in mortality in the screening group compared with the non-screening group during the entire 22-year study period. Mortality rates from all causes did not differ between the two groups $(\mathrm{P}=0.516$ for between 1987 and 2008).

In total, the study was comprised of 4,777 subjects aged between 50 and 74 years, and 327 subjects aged $>75$ years on recruitment. A stratified analysis revealed that a significant decrease in the incidence and mortality of CRC was only evident in the subgroup who were aged between 50 and 74 years $(\mathrm{P}=0.028$ and $\mathrm{P}=0.024$, respectively; Table IV). 
Table III. Incidence and mortality rates of CRC between 1987 and 2008 (per 1000 person-years).

\begin{tabular}{|c|c|c|c|c|}
\hline \multirow[b]{2}{*}{ Characteristic } & \multicolumn{2}{|c|}{ May 1987-December 2005} & \multicolumn{2}{|c|}{ May 1987-December 2008} \\
\hline & Screening & Non-screening & Screening & Non-screening \\
\hline Person-years of observation & 42881 & 13974 & 49566 & 15826 \\
\hline \multicolumn{5}{|l|}{ Colorectal cancer } \\
\hline No. of patients & 32 & 20 & 36 & 21 \\
\hline Incidence rate & 0.75 & 1.43 & 0.73 & 1.33 \\
\hline RR (95\% CI) & \multicolumn{2}{|c|}{$0.52(0.30-0.93)$} & \multicolumn{2}{|c|}{$0.55(0.32-0.95)$} \\
\hline Adjusted RR $(95 \% \mathrm{CI})^{\mathrm{a}}$ & \multicolumn{2}{|c|}{$0.50(0.29-0.88)$} & \multicolumn{2}{|c|}{$0.52(0.30-0.89)$} \\
\hline Adjusted RR $(95 \% \mathrm{CI})^{\mathrm{b}}$ & \multicolumn{2}{|c|}{$0.49(0.28-0.85)$} & \multicolumn{2}{|c|}{$0.51(0.30-0.87)$} \\
\hline \multicolumn{5}{|l|}{ Mortality from CRC } \\
\hline No. of deaths & 15 & 13 & 19 & 14 \\
\hline Mortality rate & 0.35 & 0.93 & 0.38 & 0.88 \\
\hline $\mathrm{RR}(95 \% \mathrm{CI})$ & \multicolumn{2}{|c|}{$0.38(0.18-0.81)$} & \multicolumn{2}{|c|}{$0.43(0.22-0.88)$} \\
\hline Adjusted RR $(95 \% \mathrm{CI})^{\mathrm{a}}$ & \multicolumn{2}{|c|}{$0.31(0.15-0.67)$} & \multicolumn{2}{|c|}{$0.36(0.18-0.72)$} \\
\hline Adjusted RR $(95 \% \mathrm{CI})^{\mathrm{b}}$ & \multicolumn{2}{|c|}{$0.31(0.14-0.65)$} & \multicolumn{2}{|c|}{$0.36(0.18-0.71)$} \\
\hline \multicolumn{5}{|l|}{ Mortality from all causes } \\
\hline No. of deaths & 1225 & 423 & 1607 & 530 \\
\hline Mortality rate & 28.57 & 30.27 & 32.42 & 33.49 \\
\hline RR $(95 \%$ CI $)$ & \multicolumn{2}{|c|}{$0.94(0.85-1.05)$} & \multicolumn{2}{|c|}{$0.97(0.88-1.07)$} \\
\hline
\end{tabular}

${ }^{a}$ Adjusted for age and gender. ${ }^{\mathrm{b}}$ Adjusted for age, gender, education, family history of malignant tumors, BMI, smoking status, alcohol intake, physical exercise, meat intake and aspirin use. CRC, colorectal cancer; RR, relative risk; CI, confidence interval.

Table IV. Incidence and mortality of CRC stratified by age (between May, 1987 and December, 2008).

\begin{tabular}{|c|c|c|c|c|}
\hline & \multicolumn{2}{|c|}{$50-74$ years } & \multicolumn{2}{|c|}{$\geq 75$ years } \\
\hline & Screening & Non-screening & Screening & Non-screening \\
\hline No. of subjects & 3614 & 1163 & 249 & 78 \\
\hline No. of CRC cases & $29(0.8 \%)$ & $18(1.5 \%)$ & $7(2.8 \%)$ & $3(3.8 \%)$ \\
\hline $\mathrm{RR}(95 \% \mathrm{CI})$ & \multicolumn{2}{|c|}{$0.52(0.29-0.93)$} & \multicolumn{2}{|c|}{$0.71(0.18-2.74)$} \\
\hline Adjusted RR $(95 \% \mathrm{CI})^{\mathrm{a}}$ & \multicolumn{2}{|c|}{$0.50(0.28-0.90)$} & \multicolumn{2}{|c|}{$0.69(0.18-2.67)$} \\
\hline Mortality from CRC & $14(0.4 \%)$ & $11(0.9 \%)$ & $5(2.0 \%)$ & $3(3.8 \%)$ \\
\hline $\mathrm{RR}(95 \% \mathrm{CI})$ & \multicolumn{2}{|c|}{$0.40(0.18-0.89)$} & \multicolumn{2}{|c|}{$0.43(0.10-1.83)$} \\
\hline Adjusted RR $(95 \% \mathrm{CI})^{\mathrm{a}}$ & \multicolumn{2}{|c|}{$0.36(0.16-0.80)$} & \multicolumn{2}{|c|}{$0.41(0.10-1.74)$} \\
\hline
\end{tabular}

${ }^{\mathrm{a} A d j u s t e d ~ f o r ~ a g e ~ a n d ~ g e n d e r . ~ C R C, ~ c o l o r e c t a l ~ c a n c e r ; ~ R R, ~ r e l a t i v e ~ r i s k ; ~ C I, ~ c o n f i d e n c e ~ i n t e r v a l . ~}$

Figs. 2 and 3 show the cumulative incidence and mortality of CRC, as analyzed by the Kaplan-Meier method. The differences in the cumulative incidence and mortality between the two groups was evident after the seventh and eighth years of screening, and more so thereafter.

\section{Discussion}

The current study presented a three-tier FOBT-based screening program, which has been described previously $(9,10)$. Certain cross-sectional studies for this type of combined gFOBT with FIT have demonstrated compatible sensitivity to gFOBT, but notable increased specificity $(7,10)$. The difficulty of dietary

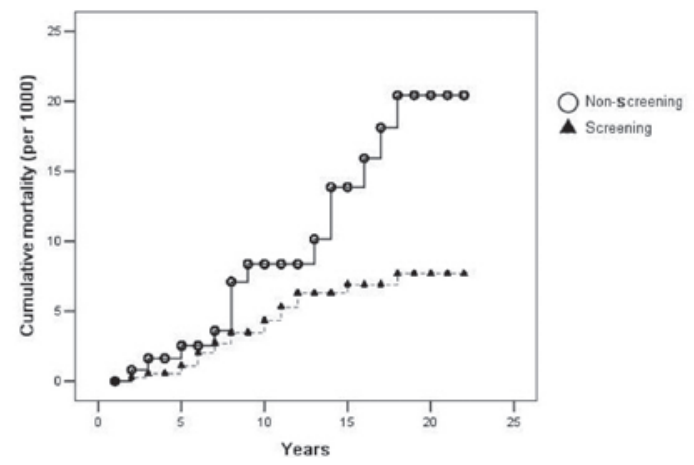

Figure 3. Cumulative mortality of colorectal cancer (CRC) in the screening and non-screening groups, by Kaplan-Meier analysis. 
restriction led to a poor specificity of guaiac-based tests in the Chinese population (11). Moreover, the advantage of using a combined test is that it reduces the cost of the FIT assay, as the FIT is only developed when the gFOBT result is positive. The observed sensitivity for CRC detection in the present study was substantially greater than that identified by Allison et al (8) (65\% for CRC) in a cross-sectional study with a similar three-tier method. This is likely to be due to the longitudinal nature of the present study, which meant that the cases missed in one screening round were identified in subsequent repeat tests.

In this 22-year longitudinal, controlled trial involving a dynamic cohort, it was demonstrated that annual FOBT-based screening resulted in a $49 \%$ decrease in colon cancer incidence and a $64 \%$ decrease in CRC-related mortality, even 3 years after the termination of the screening program. The differences in cumulative incidence and mortality were evident between the two groups after only 7 to 8 years of the study, which may indicate that the lead time of screening is $\sim 7-8$ years. To the best of our knowledge, this study is the longest follow-up of a colon cancer screening trial in a developing country. The study included a highly stable cohort with relatively few subjects lost to follow-up ( $<10 \%$ in the 22-year follow-up), but a high censoring rate due to mortality from other causes. The compliance rate was also high (almost 100\% for the first level test and $83 \%$ for colonoscopy in patients with a positive FIT result). This may be ascribed partly to bringing the screening program to a military healthcare system.

However, the study also has several limitations. The population of the study consisted only of military officials and may not consequently be representative of the general Chinese population. Additionally, the study was not randomized; the screening group consisted of subjects who chose to be screened. This may have produced a selection bias, although the screened and non-screened populations shared similar demographic features. Furthermore, there is no definite final age for the screening. Routine screening for CRC is recommended against in adults $>75$ years (12). The present data also revealed that the effect of screening on the incidence and mortality of CRC was less significant among subjects aged $>75$ years. Moreover, the sample size was relatively small compared with previous trials involving gFOBT.

Taking into account the aforementioned limitations, the present study demonstrated that a three-tier FOBT-based annual screening, even with one fecal sample, resulted in the detection of $>80 \%$ of CRCs. The study also revealed a marked decrease in CRC-related mortality (64\% after controlling potential confounding factors) compared with that which has been identified previously with gFOBT, which was in the range of 15 to $30 \%$ (4-6). The present findings were similar to the longitudinal non-controlled observational study in the Japanese population by Lee et al (13), which utilized FIT for screening and demonstrated a $70 \%$ decrease in mortality over a 13-year study period. Moreover, there are a limited number of studies demonstrating an effect of FOBT on incidence (14), while the present data revealed a $49 \%$ decrease in CRC incidence in the screening group compared with that in the non-screening group. This dramatic decrease in the incidence and mortality of CRC may be ascribed partly to the higher compliance and adenoma detection rates with subsequent resection in the present study. In the study $4 \%$ of the screening subjects were identified to have adenomas of $\geq 1 \mathrm{~cm}$ in size, while $0.8-1.7 \%$ were identified in a previous study (15). However, since the present study was not randomized, the effect of selection bias on the differences in incidence and mortality observed may not be excluded, although the two groups (screening and non-screening) exhibited similar baseline demographic characteristics. Furthermore, the grouping in the present study was according to the preference of the subjects, i.e. whether to undergo CRC screening or not. It is supposed that individuals who refuse screening have higher CRC incidence and mortality rates than those who accept testing (16). The rates of loss to follow-up were significantly higher in the non-screening group, which may indicate less benefit from the military healthcare system in the non-screening group compared with that in the screening group. All of the aforementioned factors may have contributed to the decreases in the incidence and mortality of CRC, along with the efficacy of the screening process.

In conclusion, when accepted, annual FOBT-based screening using a three-tier system in an average-risk Chinese population aged $>50$ years demonstrated $>80 \%$ sensitivity in detecting CRC. The screening significantly reduced the incidence and mortality rates of CRC. These findings suggested that annual FOBT examination coupled with a complete colonoscopy follow-up for cases with positive FOBT results is an effective approach for CRC control in China.

\section{Acknowledgements}

This study was supported by the Chinese Zonghou Medical Foundation (grant no. BJZ07).

\section{References}

1. Stewart BW and Kleihues P: World cancer report. Lyon: IARC Press: 163-166, 2003.

2. Zhang SW, Chen WQ, Kong LZ, Li LD, Lu FZ, Li GL, Meng J and Zhao P: An analysis of cancer incidence and mortality from 30 cancer registries in China, 1998 2002. Zhongguo Zhongliu 15: 430-448, 2006.

3. Levin B, Lieberman DA, McFarland B, Andrews KS, Brooks D, Bond J, Dash C, Giardiello FM, Glick S, Johnson D, Johnson CD, et al; American Cancer Society Colorectal Cancer Advisory Group; US Multi-Society Task Force; American College of Radiology Colon Cancer Committee: Screening and surveillance for the early detection of colorectal cancer and adenomatous polyps, 2008: a joint guideline from the American Cancer Society, the US Multi-Society Task Force on Colorectal Cancer, and the American College of Radiology. Gastroenterology 134: 1570-1595, 2008.

4. Mandel JS, Bond JH, Church TR, Snover DC, Bradley GM, Schuman LM and Ederer F: Reducing mortality from colorectal cancer by screening for fecal occult blood. Minnesota Colon Cancer Control Study. N Engl J Med 328: 1365-1371, 1993.

5. Kronborg O, Fenger C, Olsen J, Jorgensen OD and Søndergaard O: Randomised study of screening for colorectal cancer with faecal-occult-blood test. Lancet 348: 1467-1471, 1996.

6. Hardcastle JD, Chamberlain JO, Robinson MH, Moss SM, Amar SS, Balfour TW, James PD and Mangham CM: Randomised controlled trial of faecal-occult-blood screening for colorectal cancer. Lancet 348: 1472-1477, 1996.

7. Allison JE, Sakoda LC, Levin TR, Tucker JP, Tekawa IS, Cuff T, Pauly MP, Shlager L, Palitz AM, Zhao WK, Schwartz JS, Ransohoff DF and Selby JV: Screening for colorectal neoplasms with new fecal occult blood tests: update on performance characteristics. J Natl Cancer Inst 99: 1462-1470, 2007.

8. Allison JE, Tekawa IS, Ransom LJ and Adrain AL: A comparison of fecal occult-blood tests for colorectal-cancer screening. N Engl J Med 334: 155-159, 1996. 
9. Li S, Nie Z, Li N, Li J, Zhang P, Yang Z, Mu S, Du Y, Hu J, Yuan S, Qu H et al: Colorectal cancer screening for the natural population of Beijing with sequential fecal occult blood test: a multicenter study. Chin Med J (Engl) 116: 200-202, 2003.

10. Li S, Wang H, Hu J, Li N, Liu Y, Wu Z, Zheng Y, Wang H, Wu K, Ye H and Rao J: New immunochemical fecal occult blood test with two-consecutive stool sample testing is a cost-effective approach for colon cancer screening: Results of a prospective multicenter study in Chinese patients. Int J Cancer 118: 3078-3083, 2006.

11. Wong BC, Wong WM, Cheung KL, Tong TS, Rozen P, Young GP, Chu KW, Ho J, Law WL, Tung HM, Lai KC, Hu WH, Chan CK and Lam SK: A sensitive guaiac faecal occult blood test is less useful than an immunochemical test for colorectal cancer screening in a Chinese population. Aliment Pharmacol Ther 18: 941-946, 2003

12. Calogne N, Petitti DB, DeWitt TG, Dietrich AJ, Gregory KD, Harris R, Isham G, LeFevre ML, Leipzig RM, Loveland-Cherry C, Marion LN et al; U.S. Preventive Services Task Force: Screening for colorectal cancer: U.S. Preventie Services Task Force recommendation statement. Ann Intern Med 149: 627-637, 2008.
13. Lee KJ, Inoue M, Otani T, Iwasaki M, Sasazuki S, Tsugane S Japan Public Health Center-based Prospective Study: Colorectal cancer screening using fecal occult blood test and subsequent risk of colorectal cancer: a prospective cohort study in Japan. Cancer Detect Prev 31: 3-11, 2007.

14. Mandel JS, Church TR, Bond JH, Ederer F, Geisser MS, Mongin SJ, Snover DC and Schuman LM: The effect of fecal occult-blood screening on the incidence of colorectal cancer. N Engl J Med 343: 1603-1607, 2000.

15. van Rossum LG, van Rijn AF, Laheij RJ, van Oijen MG, Fockens P, van Krieken HH, Verbeek AL, Jansen JB and Dekker E: Random comparison of guaiac and immunochemical fecal occult blood tests for colorectal cancer in a screening population. Gastroenterology 135: 82-90, 2008.

16. Niv Y, Lev-El M, Fraser G, Abuksis G and Tamir A: Protective effect of faecal occult blood test screening for colorectal cancer: worse prognosis for screening refusers. Gut 50: 33-37, 2002. 